\title{
Analysis of a non-uniformly elliptic and nonlinear coupled parabolic-elliptic system arising in steel hardening
}

\author{
M.T. González Montesinos ${ }^{\mathrm{a}}$ and F. Ortegón Gallego $\mathrm{b}$ \\ ${ }^{a}$ Departamento de Matemática Aplicada I, Universidad de Sevilla, Avda. Reina Mercedes, s/n, 41012 \\ Sevilla, Spain; ${ }^{b}$ Departamento de Matemáticas, Facultad de Ciencias, Universidad de Cádiz, Campus del \\ Río San Pedro, 11510 Puerto Real, Spain
}

\begin{abstract}
The goal of this work is to analyse the existence of weak solutions to a coupled nonlinear parabolic-elliptic system derived from the heating industrial process of a steel workpiece, and whose unknowns are the electric potential, the magnetic vector potential and the temperature. We introduce the harmonic regime because of the different time scales related to the electric potential and the magnetic vector potential versus the temperature. This lead us to a new system of nonlinear partial differential equations.
\end{abstract}

Keywords: steel hardening; Maxwell's equations; Joule's heating; nonlinear parabolic-elliptic equations; Sobolev spaces

\section{Introduction}

This paper is devoted to study the existence of weak solutions to a system of coupled nonlinear partial differential equations modelling the industrial process of steel hardening. This process has been thoroughly analysed along the last years [5,7-11,13-15]. A complete model, including thermomechanical effects, can be seen, for instance, in $[9,10,13]$.

Our main concern is to investigate the induction-conduction model that describes the heating industrial process of a car steering rack which is made of steel (Figure 1). Because of its importance inside an automobile, a 20-year lifetime has to be assured at least by the manufacturer.

The aim of steel heating is to produce martensite, a hard and brittle steel phase transition, along the toothed part surface, which is going to be highly stressed during its mechanical lifetime, keeping the rest ductile.

Although there are different hardening surface techniques, we focus our attention on induction hardening. Thus, a copper inductor is put in contact with the rack as Figure 2 shows, and a high frequency alternating current is switch on and flows through the coil composed of the cooper inductor and the workpiece itself, creating an alternating magnetic field which in turn induces eddy currents generating heat (Joule's effect) along the toothed part and its surroundings. 


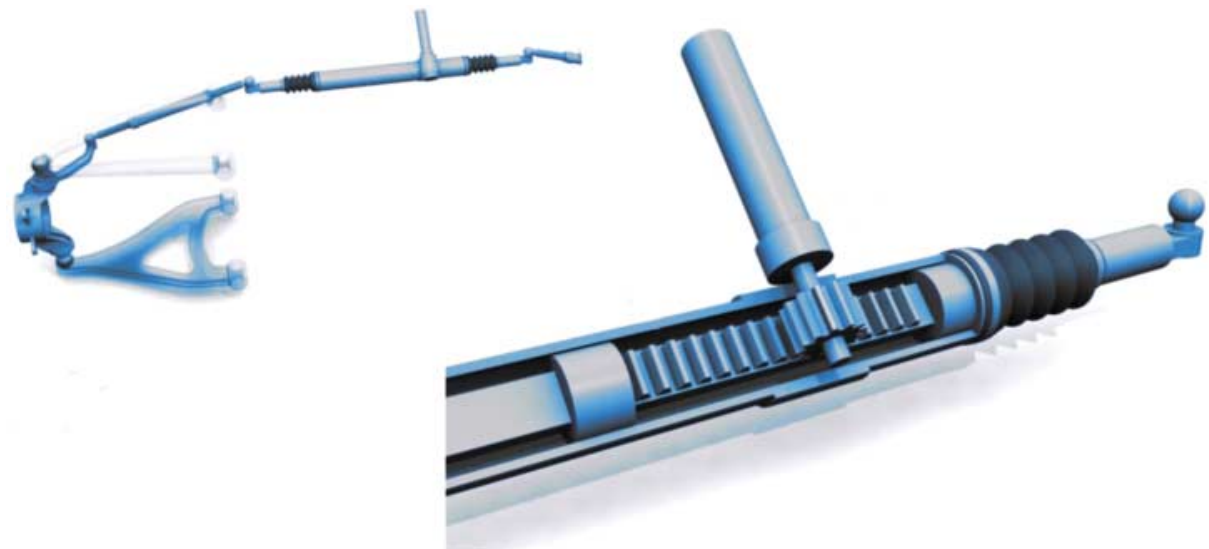

Figure 1. Characterization of an automobile steering (modified from original images at http://www.carbibles.com) (@1994-2012 Christopher J. Longhurst. All rights reserved).

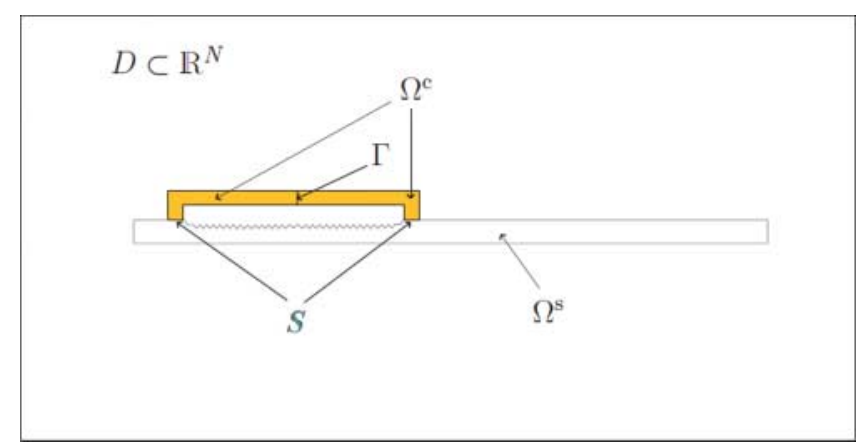

Figure 2. Domains in the problem.

After $5.5 \mathrm{~s}$, the required temperature is achieved at those parts where it is needed along the rack and the supplied electric current is turned off. Then, the workpiece is sprayed by an aqueous solution to cool it down quickly, a process called aquaquenching.

The whole industrial process are governed by a coupled nonlinear system of partial differential equations and ordinary differential equations, and a Neumann boundary condition is imposed on a fictitious cross-section $\Gamma$ in the copper inductor (Figure 2) to model the supplied current flow density.

Nevertheless, our main concern lies on the mathematical analysis of the industrial heating process described in terms of the harmonic regime. This problem was studied in [12] under more restrictive assumptions on data, but now we consider more realistic hypotheses on the electric conductivity $\sigma_{0}$, namely $\sigma_{0}$ is non-uniformly elliptic. As a result from the mathematical standpoint, we have to deal with a much more complex problem.

This work is organized as follows. Section 2 is devoted to set up the mathematical model derived from the industrial heating procedure. In Section 3, we introduce the harmonic regime associated with our problem. In Section 4, we establish the notation used along this paper and formulate some useful results. In Section 5, we give the hypotheses on data and state the existence result. Finally, in Section 6, the proof of the existence result is evolved; it is divided into three steps: setting of the approximate problems, derivation of estimates, and passing to the limit and conclusion. 


\section{The mathematical model for the heating process}

We focus on analysing the existence of weak solutions of a simplified model which does not take into account mechanical effects.

Let $\Omega, D \subset \mathrm{R}^{3}$ be bounded, connected, and Lipschitz-continuous open sets such that $\bar{\Omega} \subset$ $D, \Omega=\Omega^{\mathrm{c}} \cup \Omega^{\mathrm{s}} \cup S$ is the set of conductors, $\Omega^{\mathrm{c}}$ the copper inductor, $\Omega^{\mathrm{s}}$ the steel workpiece containing a toothed part to be hardened, $\Omega^{\mathrm{c}}$ and $\Omega^{\mathrm{s}}$ being open sets, and $S=\bar{\Omega}^{\mathrm{c}} \cap \bar{\Omega}^{\mathrm{s}}$ is the surface contact between $\Omega^{\mathrm{c}}$ and $\Omega^{\mathrm{s}}, \Omega^{\mathrm{c}} \cap \Omega^{\mathrm{s}}=\varnothing$ (Figure 2 ).

The electric displacement term may be disregarded in the set of Maxwell's equations since we deal with high frequency current density of about $80 \mathrm{kHz}$ :

$$
\begin{aligned}
\nabla \times \mathcal{E} & =-\mathcal{B}_{, t}, \\
\nabla \times \mathcal{H} & =\mathcal{J}, \\
\nabla \cdot \mathcal{B} & =0,
\end{aligned}
$$

where $\mathcal{E}$ is the electric field, $\mathcal{J}$ the current density, $\mathcal{H}$ the magnetic field, and $\mathcal{B}$ the magnetic induction. Putting $\sigma$ the electric conductivity and $\mu$ the magnetic permeability, the constitutive basic laws are given by

$$
\begin{aligned}
\mathcal{J} & =\sigma \mathcal{E}, \\
\mathcal{B} & =\mu \mathcal{H}
\end{aligned}
$$

Taking into account Equation (3), we introduce the magnetic vector potential $\mathcal{A}$ as

$$
\mathcal{B}=\nabla \times \mathcal{A}
$$

In view of Equations (1) and (6), $\nabla \times\left(\mathcal{E}+\mathcal{A}_{, t}\right)=\mathbf{0}$. Hence, there exists a scalar function $\phi$, which will be called electric potential, such that

$$
\mathcal{E}+\mathcal{A}_{, t}=-\nabla \phi
$$

and therefore

$$
\nabla \cdot[\sigma(\theta) \nabla \phi]=-\nabla \cdot\left[\sigma(\theta) \mathcal{A}_{, t}\right] .
$$

In practice, the right-hand side of this equation is neglected. Thus,

$$
\nabla \cdot[\sigma(\theta) \nabla \phi]=0 .
$$

Using Equations (2), (4)-(6) and $\mathcal{E}+\mathcal{A}_{, t}=-\nabla \phi$, the next equation is derived:

$$
\sigma_{0}(\theta) \mathcal{A}_{, t}+\nabla \times\left(\frac{1}{\mu} \nabla \times \mathcal{A}\right)-\delta \nabla(\nabla \cdot \mathcal{A})+\sigma_{0}(\theta) \nabla \phi=\mathbf{0} \quad \text { in } D_{T}=D \times(0, T),
$$

where

$$
\sigma_{0}(x, s)= \begin{cases}\sigma(x, s) & \text { if } x \in \bar{\Omega} \\ 0 & \text { otherwise }\end{cases}
$$

In [13], it is assumed the Coulomb gauge condition for the magnetic vector potential, namely, $\nabla \cdot \mathcal{A}=0$. Instead, we include a penalty term in this equation of the form $-\delta \nabla(\nabla \cdot \mathcal{A})$, where $\delta$ is a small constant. 
As far as the boundary conditions are concerned, we consider for the magnetic vector potential

$$
\mathcal{A}=\mathbf{0} \quad \text { on } \partial D \times(0, T)
$$

For the electric potential,

$$
\begin{gathered}
\sigma(\theta) \frac{\partial \phi}{\partial n}=0 \quad \text { on } \partial \Omega \times(0, T), \\
{\left[\sigma(\theta) \frac{\partial \phi}{\partial v}\right]_{\Gamma}=j_{S} \quad \text { on } \Gamma \times(0, T),}
\end{gathered}
$$

where $j_{S}$ is the external source current density, $[\cdot]_{\Gamma}$ denotes the jump across the inner surface $\Gamma$, and $v$ is a unit normal vector on $\Gamma$.

Finally, the combined effect of both conduction and induction gives rise to a production term in the energy balance equation, namely Joule's heating:

$$
\sigma(\theta)|\mathcal{E}|^{2}=\sigma(\theta)\left|\mathcal{A}_{t}+\nabla \phi\right|^{2}
$$

Here we add a source term $G$ coming from phase transitions and mechanical deformation. In our analysis, the density of the workpiece, $\rho$, and the specific heat capacity at constant strain, $c$, are taken to be constant values, and without loss of generality, we assume that $\rho c=1$. Then, the initial conditions problem for the energy balance reads as follows:

$$
\begin{aligned}
\theta_{, t}-\nabla \cdot(\kappa(\theta) \nabla \theta) & =\sigma(\theta)\left|\mathcal{A}_{, t}+\nabla \phi\right|^{2}+G \quad \text { in } \Omega_{T}=\Omega \times(0, T), \\
\frac{\partial \theta}{\partial n} & =0 \quad \text { on } \partial \Omega \times(0, T), \\
\theta(\cdot, 0) & =\theta_{0} \quad \text { in } \Omega,
\end{aligned}
$$

where $\kappa$ is the thermal conductivity, which also depends on the temperature of the steel workpiece.

\section{The harmonic regime}

Electromagnetic fields generated by high frequency currents are sinusoidal in time. Consequently, both the electric potential, $\phi$, and the magnetic vector potential, $\mathcal{A}$, take the form $[2,3,16,18]$ $\mathcal{F}(x, t)=\operatorname{Re}[\exp (\mathrm{i} \omega t) F(x)]$, where $F$ is a complex-valued function or vector field, and $\omega=2 \pi f$ is the angular frequency, $f$ being the electric current frequency. In general, $F$ also depends on $t$, but at a time scale much greater than $1 / \omega$. In this way, we may introduce the complex-valued fields $\varphi, \boldsymbol{A}$ and $\boldsymbol{j}$ as

$$
\phi=\operatorname{Re}[\exp (\mathrm{i} \omega t) \varphi(x)], \quad \mathcal{A}=\operatorname{Re}[\exp (\mathrm{i} \omega t) \boldsymbol{A}(x)], \quad j_{S}=\operatorname{Re}[\exp (\mathrm{i} \omega t) \boldsymbol{j}(x, t)] .
$$

As a far as the numerical simulation of a system like Equations (7)-(14) is concerned (see [9]), the introduction of these new variables is quite convenient since the time scale describing the evolution of both $\varphi$ and $\boldsymbol{A}$ is much smaller than that of the temperature $\theta$. In the case of steel heat treating, $f$ is of about $80 \mathrm{kHz}$.

Nevertheless, the fields $\varphi$ and $\boldsymbol{A}$ still depend on time. This dependence comes from the term $\sigma(\theta)$, with $\theta=\theta(x, t)$, and from $j(x, t)$. Moreover, the change of variables (15) is carried out by most authors (see $[2,3,16,18]$ among others) in order to perform numerical simulations. Here we analyse the existence of a solution of the model derived in this way. 
Taking into account the time dependence in the complex-valued functions, we define

$$
\phi=\operatorname{Re}[\exp (\mathrm{i} \omega t) \varphi(x, t)], \quad \mathcal{A}=\operatorname{Re}[\exp (\mathrm{i} \omega t) \boldsymbol{A}(x, t)], \quad j_{S}=\operatorname{Re}[\exp (\mathrm{i} \omega t) \boldsymbol{j}(x, t)] .
$$

Using Equation (16) in Equations (7)-(14), we obtain the so-called harmonic regime given by

$$
\begin{aligned}
& \nabla \cdot(\sigma(\theta) \nabla \varphi)=0 \quad \text { in } \Omega_{T}, \\
& \frac{\partial \varphi}{\partial n}=0 \quad \text { on } \partial \Omega \times(0, T), \\
& {\left[\sigma(\theta) \frac{\partial \varphi}{\partial v}\right]_{\Gamma}=\boldsymbol{j} \quad \text { on } \Gamma \times(0, T),} \\
& \mathrm{i} \omega \sigma_{0}(\theta) \boldsymbol{A}+\nabla \times\left(\frac{1}{\mu} \nabla \times \boldsymbol{A}\right)-\delta \nabla(\nabla \cdot \boldsymbol{A})+\sigma_{0}(\theta) \nabla \varphi=0 \quad \text { in } D_{T}, \\
& \boldsymbol{A}=\mathbf{0} \quad \text { on } \partial D \times(0, T), \\
& \theta_{, t}-\nabla \cdot(\kappa(\theta) \nabla \theta)=\frac{\sigma(\theta)}{2}|\mathrm{i} \omega \boldsymbol{A}+\nabla \varphi|^{2}+G \quad \text { in } \Omega_{T}, \\
& \frac{\partial \theta}{\partial n}=0 \quad \text { on } \partial \Omega \times(0, T), \\
& \theta(\cdot, 0)=\theta_{0} \quad \text { in } \Omega,
\end{aligned}
$$

where the unknowns $\varphi$, the electric potential, and $\boldsymbol{A}$, the magnetic vector potential, are complexvalued.

Note that the term $\sigma_{0}(\theta) \boldsymbol{A}_{, t}$ has been neglected in Equation (20) since it is expected to be a lower order term in front of $\mathrm{i} \omega \sigma_{0}(\theta) \boldsymbol{A}$. Also, in Equation (22), the production term in the energy equation $\left|\mathcal{A}_{, t}+\nabla \phi\right|^{2}$ is replaced by $|\mathrm{i} \omega \boldsymbol{A}+\nabla \varphi|^{2} / 2$, which represents the effective Joule's heating. This is an approximation of the average of $\left|\mathcal{A}_{t}+\nabla \phi\right|^{2}$ over a time period, that is,

$$
\frac{1}{\omega} \int_{t}^{t+\omega}\left|\mathcal{A}_{, t}+\nabla \phi\right|^{2} \approx \frac{1}{2}|\mathrm{i} \omega \boldsymbol{A}+\nabla \varphi|^{2} .
$$

Also, this last expression becomes an equality if $\boldsymbol{A}=\boldsymbol{A}(x)$.

\section{Some preliminary results}

From now on, for a normed space $V$, we denote $\boldsymbol{V}=V^{3}$. Also, let $H^{1}(\Omega)=\left\{v \in L^{2}(\Omega) / \nabla v \in\right.$ $\left.\boldsymbol{L}^{2}(\Omega)\right\}$ be the complex-valued usual Sobolev space and consider the Hilbert space $H_{0}^{1}(D)=\{v \in$ $H^{1}(D) / v=0$ on $\Omega$ \}. For these spaces, we have the next respective useful results.

LEMMA 1 If $\Omega$ is a bounded, connected and Lipschitz-continuous open set, the quotient space $H^{1}(\Omega) / \mathbb{C}$ provided with the norm

$$
\|\hat{u}\|_{H^{1}(\Omega) / \mathbb{C}}=\inf _{u \in \hat{u}}\|u\|_{H^{1}(\Omega)}
$$

is a Hilbert space. Moreover, the seminorm $\hat{u} \in H^{1}(\Omega) / \mathbb{C} \mapsto|u|_{H^{1}(\Omega)}$ is equivalent to norm (25), that is, there are two constant values $c_{1}, c_{2}>0$ such that, for any $\hat{u} \in H^{1}(\Omega) / \mathbb{C}$ and $u \in \hat{u}$, one has

$$
c_{1}\|\hat{u}\|_{H^{1}(\Omega) / \mathbb{C}} \leq\left(\int_{\Omega}|\nabla u|^{2}\right)^{1 / 2} \leq c_{2}\|\hat{u}\|_{H^{1}(\Omega) / \mathbb{C}} .
$$


THEOREM 1 For all $\boldsymbol{v} \in \boldsymbol{H}_{0}^{1}(D)$, we have that

$$
|\boldsymbol{v}|_{\boldsymbol{H}^{1}(D)}^{2}=\|\nabla \times v\|_{L^{2}(D)}^{2}+\|\nabla \cdot v\|_{L^{2}(D)}^{2} .
$$

This result can be found in [1].

For any $1 \leq p \leq \infty$, we consider the Banach space $W^{1, p}(\Omega)$ equipped with their standard norm, and $\left(W^{1, p}(\Omega)\right)^{\prime}$ its dual (topological and algebraic) space.

If $X$ is a Banach space, we write $L^{p}(X)=L^{p}(0, T ; X)$ and $W^{1, p}(X)=W^{1, p}(0, T ; X)$, in other words,

$$
W^{1, p}(X)=\left\{v \in L^{p}(X) / v_{, t} \in L^{p}(X)\right\},
$$

where the derivative $v_{, t}$ is taken in the sense of distributions in $(0, T)$. Both, $L^{p}(X)$ and $W^{1, p}(X)$ are Banach spaces, and $W^{1, p}(X) \hookrightarrow C([0, T] ; X)$ with continuous embedding. The following result is very well known (see [17]).

Lemma 2 Let $X, B$, and $Y$ be three Banach spaces such that $X \hookrightarrow B \hookrightarrow Y$, every embedding being continuous and the inclusion $X \hookrightarrow B$ compact. For $1 \leq p, q<+\infty$, let $\mathcal{W}$ be the Banach space defined as

$$
\mathcal{W}=\left\{v \in L^{p}(X) / v_{, t} \in L^{q}(Y)\right\} .
$$

Then, the inclusion $\mathcal{W} \hookrightarrow L^{p}(B)$ holds and is compact.

Finally, let $V$ be a close subspace of $H^{1}(\Omega)$ so that $H_{0}^{1}(\Omega) \subset V \subset H^{1}(\Omega)$ and write $\mathcal{V}=L^{2}(V)$ and $\mathcal{V}^{\prime}=L^{2}\left(V^{\prime}\right)$. Throughout this paper, $C$ stands for a generic positive constant value independent of the subscript $k$ associated with the approximate problems stated below.

\section{Hypotheses on data and the existence result}

We consider the following hypotheses on data of system (17)-(24):

(H.1) $\sigma_{0}: D \times \mathrm{R} \mapsto \mathrm{R}$ is given by

$$
\sigma_{0}(x, s)= \begin{cases}\sigma_{\mathrm{s}}(s) & \text { if } x \in \Omega^{\mathrm{s}}, s \in \mathrm{R}, \\ \sigma_{\mathrm{c}}(s) & \text { if } x \in \Omega^{\mathrm{c}}, s \in \mathrm{R}, \\ 0 & \text { if } x \in D \backslash \bar{\Omega}, s \in \mathrm{R},\end{cases}
$$

where $\sigma=\left.\sigma_{0}\right|_{\Omega \times \mathrm{R}}, \sigma_{\mathrm{s}}, \sigma_{\mathrm{c}} \in C(\mathrm{R})$, and there exist some constant values $C_{1}, C_{2}, K_{1}, K_{2}>0$ such that, for some $0<\alpha<\frac{15}{7}$ and for all $s \in \mathrm{R}$,

$$
\begin{aligned}
& 0<\frac{C_{1}}{1+|s|^{\alpha}} \leq \sigma_{\mathrm{s}}(s) \leq C_{2}, \\
& 0<K_{1} \leq \sigma_{\mathrm{c}}(s) \leq K_{2} .
\end{aligned}
$$

(H.2) $\kappa: \Omega \times R \mapsto R$ is a Carathéodory function and there exist some constant values $\kappa_{1}, \kappa_{2}$ such that, almost everywhere $x \in \Omega$ and for all $s \in \mathrm{R}$,

$$
0<\kappa_{1} \leq \kappa(x, s) \leq \kappa_{2}
$$

(H.3) $\boldsymbol{j} \in L^{2}\left(H^{-1 / 2}(\Gamma)\right)$ and $\langle\boldsymbol{j}(t), 1\rangle_{\Gamma}=0$, almost everywhere $t \in(0, T)$, where $\langle\cdot, \cdot\rangle_{\Gamma}=0$ stands for the duality pair between $H^{1 / 2}(\Gamma)$ and $H^{-1 / 2}(\Gamma)$. 
(H.4) $\mu \in L^{\infty}(D)$ and there is a constant value $\mu_{*}$ so that $0<\mu_{*} \leq \mu$ in $D$.

(H.5) $G \in L^{1}\left(\Omega_{T}\right)$.

(H.6) $\theta_{0} \in L^{1}(\Omega)$.

Remark 1 In view of Equation (26), we cannot expect in general the regularity $\varphi(\cdot, t) \in H^{1}(\Omega)$. Furthermore, this hypothesis on $\sigma_{\mathrm{s}}$ has effect on $\boldsymbol{A}$ as well.

In spite of the difficulties in the analysis of system (17)-(24) derived from this assumption, we are interested in dealing with this problem because it corresponds to real cases and numerical simulations as $\alpha=2$.

Remark 2 In practice, the magnetic permeability is given by

$$
\mu(x)=\mu_{1} \chi_{\Omega^{\mathrm{s}}}+\mu_{2} \chi_{\Omega^{\mathrm{c}}}+\mu_{3} \chi_{D \backslash \Omega},
$$

where $\mu_{i}, 1 \leq i \leq 3$, are constant values such that $0<\mu_{2}<\mu_{3} \ll \mu_{1}$.

Now we state the main result.

THEOREM 2 Under the assumptions (H.1)-(H.6), there exist three measurable functions $\varphi, \theta$ : $\Omega_{T} \mapsto \mathrm{R}, \boldsymbol{A}: D_{T} \mapsto \mathrm{R}^{3}$, and a Radon measure $\boldsymbol{\mu} \in \mathcal{M}\left(\Omega_{T}\right)$ such that

$$
\begin{aligned}
& \varphi \in L^{r}\left(W^{1, r}(\Omega)\right), \quad \sigma(\theta)^{1 / 2} \nabla \varphi \in L^{\infty}\left(\boldsymbol{L}^{2}(\Omega)\right), \\
& \int_{\Omega} \sigma(\theta) \nabla \varphi \cdot \nabla \bar{v}+\langle\boldsymbol{j}, \bar{v}\rangle_{\Gamma}=0, \quad \text { for all } v \in H^{1}(\Omega) / \mathbb{C} \text { and a.e. } t \in(0, T) ; \\
& \boldsymbol{A} \in L^{\infty}\left(\boldsymbol{H}_{0}^{1}(D)\right), \\
& \mathrm{i} \omega \int_{\Omega} \sigma(\theta) \boldsymbol{A} \cdot \overline{\boldsymbol{v}}+\int_{D} \frac{1}{\mu} \nabla \times \boldsymbol{A} \cdot \nabla \times \overline{\boldsymbol{v}}+\delta \int_{D} \nabla \cdot \boldsymbol{A} \nabla \cdot \overline{\boldsymbol{v}}+\int_{\Omega} \sigma(\theta) \nabla \varphi \cdot \overline{\boldsymbol{v}}=0, \\
& \quad \text { for all } \boldsymbol{v} \in \boldsymbol{H}_{0}^{1}(D) \text { and a.e. } t \in(0, T) ;
\end{aligned}
$$

$\theta \in L^{p}\left(W^{1, p}(\Omega)\right) \cap C\left([0, T] ;\left(W^{1, p^{\prime}}(\Omega)\right)^{\prime}\right), \quad$ for all $p \in\left[1, \frac{5}{4}\right)$,

$$
\theta(\cdot, 0)=\theta_{0} \quad \text { in } \Omega
$$

$-\int_{\Omega_{T}} \theta \zeta_{, t}+\int_{\Omega_{T}} \kappa(\theta) \nabla \theta \nabla \zeta=\int_{\Omega_{T}}\left[\frac{\sigma(\theta)}{2}|\mathrm{i} \omega \boldsymbol{A}+\nabla \phi|^{2}+G\right] \zeta+\int_{\Omega_{T}} \zeta \mathrm{d} \boldsymbol{\mu}+\int_{\Omega} \theta_{0}(x) \zeta(x, 0)$, for all $\zeta \in \mathcal{D}\left(\bar{\Omega}_{T}\right)$ such that $\zeta(\cdot, T)=0$ in $\Omega$.

\section{Proof of the main result}

Along this section, we develop the proof of the existence result given in Theorem 2. To do so, we first introduce a sequence of approximate problems, then derive some estimates and finally pass to the limit. 


\subsection{Approximate problems}

For each $k \in \mathrm{N}$, we define the approximate function of $\sigma_{0}$ as

$$
\sigma_{k}(x, s)= \begin{cases}\sigma_{\mathrm{s}}(s)+\frac{1}{k} & \text { if } x \in \Omega^{\mathrm{s}}, s \in \mathrm{R}, \\ \sigma_{\mathrm{c}}(s) & \text { if } x \in \Omega^{\mathrm{c}}, s \in \mathrm{R}, \\ 0 & \text { if } x \in D \backslash \bar{\Omega}, s \in \mathrm{R} .\end{cases}
$$

We also consider a sequence $\left(\boldsymbol{j}_{k}\right) \subset C\left([0, T], H^{-1 / 2}(\Gamma)\right)$ such that

$$
\boldsymbol{j}_{k} \rightarrow \boldsymbol{j} \text { strongly in } L^{2}\left(H^{-1 / 2}(\Gamma)\right) \text {. }
$$

Then, the approximate problem of Equations (17)-(24) is given by

$$
\begin{aligned}
& \nabla \cdot\left(\sigma_{k}\left(\theta_{k}\right) \nabla \varphi_{k}\right)=0 \quad \text { in } \Omega_{T}, \\
& \frac{\partial \varphi_{k}}{\partial n}=0 \quad \text { on } \partial \Omega \times(0, T), \\
& {\left[\sigma_{k}\left(\theta_{k}\right) \frac{\partial \varphi_{k}}{\partial \nu}\right]_{\Gamma}=\boldsymbol{j}_{k} \quad \text { on } \Gamma \times(0, T),} \\
& \mathrm{i} \omega \sigma_{k}\left(\theta_{k}\right) \boldsymbol{A}_{k}+\nabla \times\left(\frac{1}{\mu} \nabla \times \boldsymbol{A}_{k}\right)-\delta \nabla\left(\nabla \cdot \boldsymbol{A}_{k}\right)+\sigma_{k}\left(\theta_{k}\right) \nabla \varphi_{k}=0 \quad \text { in } D_{T}, \\
& \boldsymbol{A}_{k}=0 \quad \text { on } \partial D \times(0, T), \\
& \theta_{k, t}-\nabla \cdot\left(\kappa\left(\theta_{k}\right) \nabla \theta_{k}\right)=F_{k} \quad \text { in } \Omega_{T}, \\
& \frac{\partial \theta_{k}}{\partial n}=0 \quad \text { on } \partial \Omega \times(0, T), \\
& \theta_{k}(\cdot, 0)=T_{k}\left(\theta_{0}\right) \quad \text { in } \Omega,
\end{aligned}
$$

where the sequence of functions $\left(F_{k}\right) \subset L^{\infty}\left(\Omega_{T}\right)$ is defined as

$$
F_{k}=T_{k}\left(\frac{1}{2} \sigma_{k}\left(\theta_{k}\right)\left|\mathrm{i} \omega \boldsymbol{A}_{k}+\nabla \varphi_{k}\right|^{2}\right)+T_{k}(G) .
$$

The variational formulation of the approximate problem (36)-(43) is

$$
\begin{aligned}
& \int_{\Omega} \sigma_{k}\left(\theta_{k}\right) \nabla \varphi_{k} \cdot \nabla \bar{\psi}+\int_{\Gamma} \boldsymbol{j}_{k} \bar{\psi}=0, \quad \text { for all } \psi \in H^{1}(\Omega) / \mathbb{C} \text { and a.e. } t \in(0, T), \\
& \mathrm{i} \omega \int_{\Omega} \sigma_{k}\left(\theta_{k}\right) \boldsymbol{A}_{k} \cdot \overline{\boldsymbol{v}}+\int_{D} \frac{1}{\mu} \nabla \times \boldsymbol{A}_{k} \cdot \nabla \times \overline{\boldsymbol{v}}+\delta \int_{D} \nabla \cdot \boldsymbol{A}_{k} \nabla \cdot \overline{\boldsymbol{v}}+\int_{\Omega} \sigma_{k}\left(\theta_{k}\right) \nabla \varphi_{k} \cdot \overline{\boldsymbol{v}}=0, \\
& \quad \text { for all } \boldsymbol{v} \in \boldsymbol{H}_{0}^{1}(D) \text { and a.e. } t \in(0, T), \\
& \int_{0}^{t}\left\langle\theta_{k, t}, v\right\rangle_{V^{\prime}, V}+\int_{0}^{t} \int_{\Omega} \kappa\left(\theta_{k}\right) \nabla \theta_{k} \nabla v=\int_{0}^{t} \int_{\Omega} F_{k} v, \quad \text { for all } v \in \mathcal{V} \text { and } t \in[0, T], \\
& \theta_{k}(\cdot, 0)=T_{k}\left(\theta_{0}\right) .
\end{aligned}
$$

It can be seen in [12] that there exists a weak solution $\left(\varphi_{k}, \boldsymbol{A}_{k}, \theta_{k}\right)$ to the system (36)-(43) such that

$$
\begin{aligned}
& \varphi_{k} \in L^{\infty}\left(H^{1}(\Omega) / \mathbb{C}\right), \quad \sigma_{k}\left(\theta_{k}\right) \nabla \varphi_{k} \in L^{\infty}\left(\boldsymbol{L}^{2}(\Omega)\right), \\
& \boldsymbol{A}_{k} \in L^{\infty}\left(\boldsymbol{H}_{0}^{1}(D)\right), \\
& \theta_{k} \in \mathcal{V} \cap C\left([0, T] ; L^{2}(\Omega)\right), \quad \theta_{k, t} \in \mathcal{V}^{\prime} .
\end{aligned}
$$




\subsection{A priori estimates}

Choosing $\psi=\varphi_{k}$ as a test function in Equation (36) and taking into account Equation (26), we have, almost everywhere $t \in(0, T)$, that

$$
\begin{aligned}
\int_{\Omega} \sigma_{k}\left(\theta_{k}\right)\left|\nabla \varphi_{k}\right|^{2} & =-\left\langle\boldsymbol{j}_{k} \bar{\varphi}_{k}\right\rangle \leq\left\|\boldsymbol{j}_{k}\right\|_{H^{-1 / 2}(\Gamma)}\left\|\varphi_{k}\right\|_{H^{1 / 2}(\Gamma)} \leq C\left\|\boldsymbol{j}_{k}\right\|_{H^{-1 / 2}(\Gamma)}\left\|\varphi_{k}\right\|_{H^{1}\left(\Omega^{\mathrm{c}}\right) / \mathbb{C}} \\
& =C\left\|\boldsymbol{j}_{k}\right\|_{H^{-1 / 2}(\Gamma)}\left(\int_{\Omega^{\mathrm{c}}}\left|\nabla \varphi_{k}\right|^{2}\right)^{1 / 2} \\
& =C\left\|\boldsymbol{j}_{k}\right\|_{H^{-1 / 2}(\Gamma)}\left(\int_{\Omega^{\mathrm{c}}} \sigma_{k}\left(\theta_{k}\right)^{-1} \sigma_{k}\left(\theta_{k}\right)\left|\nabla \varphi_{k}\right|^{2}\right)^{1 / 2} \\
& =C\left\|\boldsymbol{j}_{k}\right\|_{H^{-1 / 2}(\Gamma)}\left(\int_{\Omega^{\mathrm{c}}} \sigma_{\mathrm{c}}\left(\theta_{k}\right)^{-1} \sigma_{\mathrm{c}}\left(\theta_{k}\right)\left|\nabla \varphi_{k}\right|^{2}\right)^{1 / 2} \\
& \leq \frac{C}{K_{1}^{1 / 2}}\left\|\boldsymbol{j}_{k}\right\|_{H^{-1 / 2}(\Gamma)}\left(\int_{\Omega} \sigma_{k}\left(\theta_{k}\right)\left|\nabla \varphi_{k}\right|^{2}\right)^{1 / 2} \\
& \leq \frac{C^{2}}{2 K_{1}}\left\|\boldsymbol{j}_{k}\right\|_{H^{-1 / 2}(\Gamma)}^{2}+\frac{1}{2} \int_{\Omega} \sigma_{k}\left(\theta_{k}\right)\left|\nabla \varphi_{k}\right|^{2} .
\end{aligned}
$$

Thus, using Equation (35) and taking into account that $C\left([0, T], H^{-1 / 2}(\Gamma)\right) \subset L^{\infty}\left(H^{-1 / 2}(\Gamma)\right)$, it yields that

$$
\left(\sigma_{k}\left(\theta_{k}\right)^{1 / 2} \nabla \varphi_{k}\right) \text { is bounded in } L^{\infty}\left(\boldsymbol{L}^{2}(\Omega)\right) .
$$

With regard to the magnetic vector potential, multiplying Equation (45) by $1-i$, one has

$$
\begin{aligned}
& \omega \int_{\Omega} \sigma_{k}\left(\theta_{k}\right) \boldsymbol{A}_{k} \cdot \overline{\boldsymbol{v}}+\int_{D} \frac{1}{\mu} \nabla \times \boldsymbol{A}_{k} \cdot \nabla \times \overline{\boldsymbol{v}}+\delta \int_{D} \nabla \cdot \boldsymbol{A}_{k} \nabla \cdot \overline{\boldsymbol{v}}+\int_{\Omega} \sigma_{k}\left(\theta_{k}\right) \nabla \varphi_{k} \cdot \overline{\boldsymbol{v}} \\
& \quad+\mathrm{i}\left(\omega \int_{\Omega} \sigma_{k}\left(\theta_{k}\right) \boldsymbol{A}_{k} \cdot \overline{\boldsymbol{v}}-\int_{D} \frac{1}{\mu} \nabla \times \boldsymbol{A}_{k} \cdot \nabla \times \overline{\boldsymbol{v}}-\delta \int_{D} \nabla \cdot \boldsymbol{A}_{k} \nabla \cdot \overline{\boldsymbol{v}}\right) \\
& \quad-\mathrm{i}\left(\int_{\Omega} \sigma_{k}\left(\theta_{k}\right) \nabla \varphi_{k} \cdot \overline{\boldsymbol{v}}\right)=0, \quad \text { a.e. } t \in(0, T) .
\end{aligned}
$$

Taking $\boldsymbol{v}=\boldsymbol{A}_{k} \in L^{\infty}\left(\boldsymbol{H}_{0}^{1}(D)\right)$ as a test function, one has, almost everywhere $t \in(0, T)$,

$$
\begin{aligned}
R= & \omega \int_{\Omega} \sigma_{k}\left(\theta_{k}\right)\left|\boldsymbol{A}_{k}\right|^{2}+\int_{D} \frac{1}{\mu}\left|\nabla \times \boldsymbol{A}_{k}\right|^{2}+\delta \int_{D}\left|\nabla \cdot \boldsymbol{A}_{k}\right|^{2}+\int_{\Omega} \sigma_{k}\left(\theta_{k}\right) \nabla \varphi_{k} \cdot \overline{\boldsymbol{A}}_{k} \\
& +\mathrm{i}\left(\omega \int_{\Omega} \sigma_{k}\left(\theta_{k}\right)\left|\boldsymbol{A}_{k}\right|^{2}-\int_{D} \frac{1}{\mu}\left|\nabla \times \boldsymbol{A}_{k}\right|^{2}-\delta \int_{D}\left|\nabla \cdot \boldsymbol{A}_{k}\right|^{2}-\int_{\Omega} \sigma_{k}\left(\theta_{k}\right) \nabla \varphi_{k} \cdot \overline{\boldsymbol{A}}_{k}\right)=0
\end{aligned}
$$

whence $\operatorname{Re}(R)=0$, that is,

$$
\begin{aligned}
& \omega \int_{\Omega} \sigma_{k}\left(\theta_{k}\right)\left|\boldsymbol{A}_{k}\right|^{2}+\int_{D} \frac{1}{\mu}\left|\nabla \times \boldsymbol{A}_{k}\right|^{2}+\delta \int_{D_{T}}\left|\nabla \cdot \boldsymbol{A}_{k}\right|^{2} \\
& \quad+\int_{\Omega} \sigma_{k}\left(\theta_{k}\right)\left[\operatorname{Re}\left(\nabla \varphi_{k} \cdot \overline{\boldsymbol{A}}_{k}\right)+\operatorname{Im}\left(\nabla \varphi_{k} \cdot \overline{\boldsymbol{A}}_{k}\right)\right]=0, \quad \text { a.e. } t \in(0, T) .
\end{aligned}
$$


Owing to (H.1) and (H.3) and applying Young's inequality, we have, almost everywhere in $(0, T)$,

$$
\begin{aligned}
& \omega \int_{\Omega} \sigma_{k}\left(\theta_{k}\right)\left|\boldsymbol{A}_{k}\right|^{2}+\|\mu\|_{L^{\infty}\left(D_{T}\right)}^{-1} \int_{D}\left|\nabla \times \boldsymbol{A}_{k}\right|^{2}+\delta \int_{D}\left|\nabla \cdot \boldsymbol{A}_{k}\right|^{2} \\
& \quad \leq \omega \int_{\Omega} \sigma_{k}\left(\theta_{k}\right)\left|\boldsymbol{A}_{k}\right|^{2}+\int_{D} \frac{1}{\mu}\left|\nabla \times \boldsymbol{A}_{k}\right|^{2}+\delta \int_{D}\left|\nabla \cdot \boldsymbol{A}_{k}\right|^{2} \\
& \quad=-\int_{\Omega} \sigma_{k}\left(\theta_{k}\right)\left[\operatorname{Re}\left(\nabla \varphi_{k} \cdot \overline{\boldsymbol{A}}_{k}\right)+\operatorname{Im}\left(\nabla \varphi_{k} \cdot \overline{\boldsymbol{A}}_{k}\right)\right] \\
& \quad \leq 2 \int_{\Omega} \sigma_{k}\left(\theta_{k}\right)\left|\nabla \varphi_{k}\right|\left|\boldsymbol{A}_{k}\right|=2 \int_{\Omega} \sigma_{k}\left(\theta_{k}\right)^{1 / 2}\left|\boldsymbol{A}_{k}\right| \sigma_{k}\left(\theta_{k}\right)^{1 / 2}\left|\nabla \varphi_{k}\right| \\
& \quad \leq \frac{\omega}{2} \int_{\Omega} \sigma_{k}\left(\theta_{k}\right)\left|\boldsymbol{A}_{k}\right|^{2}+\frac{2}{\omega} \int_{\Omega} \sigma_{k}\left(\theta_{k}\right)\left|\nabla \varphi_{k}\right|^{2} .
\end{aligned}
$$

Defining $K=\min \left(\omega / 2,\|\mu\|_{L^{\infty}\left(D_{T}\right)}^{-1}, \delta\right)$, we finally deduce that

$$
\int_{\Omega} \sigma_{k}\left(\theta_{k}\right)\left|\boldsymbol{A}_{k}\right|^{2}+\int_{D}\left|\nabla \times \boldsymbol{A}_{k}\right|^{2}+\int_{D}\left|\nabla \cdot \boldsymbol{A}_{k}\right|^{2} \leq \frac{2}{\omega K} \int_{\Omega} \sigma_{k}\left(\theta_{k}\right)\left|\nabla \varphi_{k}\right|^{2} \quad \text { a.e. } t \in(0, T),
$$

wherefrom, according to Equation (48), it yields

$$
\left(\boldsymbol{A}_{k}\right) \text { is bounded in } L^{\infty}\left(\boldsymbol{H}_{0}^{1}(D)\right) \text {. }
$$

From Equations (48) and (49), it is straightforward that

$$
\left(F_{k}\right) \text { is bounded in } L^{\infty}\left(L^{1}(\Omega)\right) \text {. }
$$

As far as the temperature is concerned, owing to Equation (50) and (H.5), we may show that

$$
\left(\theta_{k}\right) \text { is bounded in } L^{p}\left(W^{1, p}(\Omega)\right), \quad \text { for all } p \in\left[1, \frac{5}{4}\right) .
$$

Remark 3 A proof of Equation (51) can be found in [4] for homogeneous Dirichlet boundary conditions. Later, Clain [6] extended this result to the case of homogeneous Neumann boundary conditions. Using the same procedures attained in these works, it can be shown that Equation (51) also holds in our context.

Owing to (H.2) and Equation (51), $\left(\kappa\left(\theta_{k}\right) \nabla \theta_{k}\right)$ is bounded in $L^{p}\left(\boldsymbol{L}^{p}(\Omega)\right)$, wherefrom $(\nabla$. $\left.\left(\kappa\left(\theta_{k}\right) \nabla \theta_{k}\right)\right)$ is bounded in $L^{1}\left(\left(W^{1, p^{\prime}}(\Omega)\right)^{\prime}\right)$. Since $N=3$, the Sobolev embedding implies that $L^{1}(\Omega) \subset\left(W^{1, q}(\Omega)\right)^{\prime}$, for all $q>3$. Moreover, $p<\frac{5}{4}$ implies $p^{\prime}>5$. Specifically, $L^{1}(\Omega) \subset$ $\left(W^{1, p^{\prime}}(\Omega)\right)^{\prime}$ for all $p \in\left[1, \frac{5}{4}\right)$, and the next continuous embedding holds:

$$
L^{1}(\Omega)+\left(W^{1, p^{\prime}}(\Omega)\right)^{\prime} \hookrightarrow\left(W^{1, p^{\prime}}(\Omega)\right)^{\prime}, \quad \text { with } 1 \leq p<\frac{5}{4},
$$

and then

$$
\left(\frac{\mathrm{d} \theta_{k}}{\mathrm{~d} t}\right) \text { is bounded in } L^{1}\left(\left(W^{1, p^{\prime}}(\Omega)\right)^{\prime}\right), \quad \text { for all } p \in\left[1, \frac{5}{4}\right)
$$




\subsection{Passing to the limit}

Let $1 \leq q<p^{*}=3 p /(3-p)$ and put $X=W^{1, p}(\Omega), B=L^{q}(\Omega)$, and $Y=\left(W^{1, p^{\prime}}(\Omega)\right)^{\prime}$. The embeddings $X \hookrightarrow B$ and $B \hookrightarrow Y$ are compact and continuous, respectively, so Lemma 2 implies that the Banach space

$$
\mathcal{W}=\left\{v \in L^{p}\left(W^{1, p}(\Omega)\right) / v_{, t} \in L^{1}\left(\left(W^{1, p^{\prime}}(\Omega)\right)^{\prime}\right)\right\}
$$

is such that the embedding $\mathcal{W} \hookrightarrow L^{p}\left(L^{q}(\Omega)\right)$ holds and is compact. On the other hand, if $1 \leq p<$ $\frac{5}{4}$, then $1 \leq q<\frac{15}{7}$, and consequently, owing to Equations (51) and (52),

$$
\left(\theta_{k}\right) \text { is relatively compact in } L^{p}\left(L^{q}(\Omega)\right) \text { for all } 1 \leq p<\frac{5}{4}, 1 \leq q<\frac{15}{7},
$$

whereof there exists a function $\theta \in L^{p}\left(L^{q}(\Omega)\right)$ such that, up to a subsequence,

$$
\theta_{k} \rightarrow \theta \text { strongly in } L^{p}\left(L^{q}(\Omega)\right) \text { and a.e. in } \Omega_{T} .
$$

Moreover, Equations (51) and (52) lead us to

$$
\theta \in C\left([0, T] ;\left(W^{1, p^{\prime}}(\Omega)\right)^{\prime}\right),
$$

and the initial condition (24) makes sense at least in the space $\left(W^{1, p^{\prime}}(\Omega)\right)^{\prime}$.

On the other hand, $\left(\sigma_{k}\left(\theta_{k}\right)\right)$ is bounded in $L^{\infty}\left(\Omega_{T}\right)$, so from (H.1) and Equation (54) there is a subsequence, denoted in the same way, such that

$$
\sigma_{k}\left(\theta_{k}\right) \rightarrow \sigma(\theta) \text { weakly-* in } L^{\infty}\left(\Omega_{T}\right) \text { and a.e. in } \Omega_{T} .
$$

Also, (H.1) and Equation (53) imply that

$$
\left(\sigma_{k}\left(\theta_{k}\right)^{-1}\right) \text { is bounded in } L^{p}\left(L^{q / \alpha}(\Omega)\right) .
$$

Furthermore, by virtue of the continuity of the functions $1 / \sigma_{\mathrm{s}}$ and $1 / \sigma_{\mathrm{c}}$ and Equation (54), we obtain that

$$
\sigma_{k}\left(\theta_{k}\right)^{-1} \rightarrow \sigma(\theta)^{-1} \text { a.e. in } \Omega_{T} .
$$

Consequently, choosing $\alpha \leq q$ and $m<\min (p, q / \alpha)$, we deduce, up to a subsequence,

$$
\sigma_{k}\left(\theta_{k}\right)^{-1} \rightarrow \sigma(\theta)^{-1} \text { strongly in } L^{m}\left(\Omega_{T}\right) \text {. }
$$

Now we establish an estimate on $\left(\varphi_{k}\right)$ in $L^{r}\left(W^{1, r}(\Omega)\right)$, for some $1<r<2$. Indeed, in view of Equations (26), (27), and (48), and applying Hölder's inequality, one has

$$
\begin{aligned}
\int_{\Omega}\left|\nabla \varphi_{k}\right|^{r} & =\int_{\Omega} \sigma_{k}\left(\theta_{k}\right)^{-r / 2} \sigma_{k}\left(\theta_{k}\right)^{r / 2}\left|\nabla \varphi_{k}\right|^{r} \\
& \leq\left(\int_{\Omega} \sigma_{k}\left(\theta_{k}\right)^{r /(r-2)}\right)^{(2-r) / 2}\left(\int_{\Omega} \sigma_{k}\left(\theta_{k}\right)\left|\nabla \varphi_{k}\right|^{2}\right)^{r / 2} \leq C\left(\int_{\Omega} \sigma_{k}\left(\theta_{k}\right)^{r /(r-2)}\right)^{(2-r) / 2} \\
& =C\left(\int_{\Omega^{\mathrm{s}}} \sigma_{k}\left(\theta_{k}\right)^{r /(r-2)}+\int_{\Omega^{\mathrm{c}}} \sigma_{k}\left(\theta_{k}\right)^{r /(r-2)}\right)^{(2-r) / 2} \\
& \leq C\left[\frac{1}{C_{1}^{r /(2-r)}} \int_{\Omega^{\mathrm{s}}}\left(1+\left|\theta_{k}\right|^{\alpha}\right)^{r /(2-r)}+\left|\Omega^{\mathrm{c}}\right| K_{1}^{r /(r-2)}\right]^{(2-r) / 2} \\
& \leq C\left[1+\left(\int_{\Omega}\left|\theta_{k}\right|^{\alpha r /(2-r)}\right)^{(2-r) / 2}\right] .
\end{aligned}
$$


In view of Equation (53), we can choose $1<r<2$ and $0<\alpha<\frac{15}{7}$, and one holds that

$$
\left(\varphi_{k}\right) \text { is bounded in } L^{r}\left(W^{1, r}(\Omega)\right),
$$

which implies the existence of a function $\varphi \in L^{r}\left(W^{1, r}(\Omega)\right)$ such that, up to a subsequence,

$$
\varphi_{k} \rightarrow \varphi \text { weakly in } L^{r}\left(W^{1, r}(\Omega)\right) .
$$

Owing to Equation (48), there is a function $\Phi \in L^{\infty}\left(\boldsymbol{L}^{2}(\Omega)\right)$ so that, for some subsequence,

$$
\sigma_{k}\left(\theta_{k}\right)^{1 / 2} \nabla \varphi_{k} \rightarrow \Phi \text { weakly-* in } L^{\infty}\left(\boldsymbol{L}^{2}(\Omega)\right) .
$$

But Equations (56) and (61) also implies that

$$
\sigma_{k}\left(\theta_{k}\right)^{1 / 2} \nabla \varphi_{k} \rightarrow \sigma(\theta)^{1 / 2} \nabla \varphi \text { weakly in } L^{r}\left(\boldsymbol{L}^{r}(\Omega)\right),
$$

and so $\Phi=\sigma(\theta)^{1 / 2} \nabla \varphi \in L^{\infty}\left(\boldsymbol{L}^{2}(\Omega)\right)$.

On the other hand, making $k \rightarrow \infty$ in Equation (44), one has that, almost everywhere $t \in(0, T)$,

$$
\begin{aligned}
\int_{\Omega} \sigma_{k}\left(\theta_{k}\right) \nabla \varphi_{k} \cdot \nabla \bar{\psi} & \rightarrow \int_{\Omega} \sigma(\theta) \nabla \varphi \cdot \nabla \bar{\psi} \\
\int_{\Gamma} \boldsymbol{j}_{k} \bar{\psi} & \rightarrow \int_{\Gamma} \boldsymbol{j} \bar{\psi}
\end{aligned}
$$

wherefrom

$$
\int_{\Omega} \sigma(\theta) \nabla \varphi \cdot \nabla \bar{\psi}=-\int_{\Gamma} j \bar{\psi}, \quad \text { a.e. } t \in(0, T) \text { and for all } \psi \in H^{1}(\Omega) / \mathbb{C} .
$$

From Equation (49) there exists a function $\boldsymbol{A} \in L^{\infty}\left(\boldsymbol{H}_{0}^{1}(D)\right)$ such that, for suitable subsequences,

$$
\begin{aligned}
\sigma_{k}\left(\theta_{k}\right) \boldsymbol{A}_{k} & \rightarrow \sigma(\theta) \boldsymbol{A} \text { weakly- } * \text { in } L^{\infty}\left(\boldsymbol{L}^{2}(\Omega)\right), \\
\boldsymbol{A}_{k} & \rightarrow \boldsymbol{A} \text { weakly-* in } L^{\infty}\left(\boldsymbol{H}_{0}^{1}(D)\right) .
\end{aligned}
$$

Making $k \rightarrow \infty$ in Equation (45), we obtain

$$
\begin{aligned}
& \mathrm{i} \omega \int_{\Omega} \sigma(\theta) \boldsymbol{A} \cdot \overline{\boldsymbol{v}}+\int_{D} \frac{1}{\mu} \nabla \times \boldsymbol{A} \cdot \nabla \times \overline{\boldsymbol{v}}+\delta \int_{D} \nabla \cdot \boldsymbol{A} \nabla \cdot \overline{\boldsymbol{v}}+\int_{\Omega} \sigma(\theta) \nabla \varphi \cdot \overline{\boldsymbol{v}}=0, \\
& \quad \text { for all } \boldsymbol{v} \in \boldsymbol{H}_{0}^{1}(D) \text { and a.e. } t \in(0, T) .
\end{aligned}
$$

Finally, from the convergences achieved till now we cannot assure the strong convergence of Equation (50), and it is only known that there exists a Radon measure $\boldsymbol{\mu} \in \mathcal{M}\left(\Omega_{T}\right)$ such that, up to a subsequence,

$$
\begin{aligned}
F_{k}= & T_{k}\left(\frac{1}{2} \sigma_{k}\left(\theta_{k}\right)\left|\mathrm{i} \omega \boldsymbol{A}_{k}+\nabla \varphi_{k}\right|^{2}\right)+T_{k}(G) \rightarrow \frac{1}{2} \sigma(\theta)|\mathrm{i} \omega \boldsymbol{A}+\nabla \varphi|^{2} \\
& +G+\boldsymbol{\mu} \text { weakly-* in } \mathcal{M}\left(\Omega_{T}\right) .
\end{aligned}
$$

Using the convergences (54), (56), (61), (62), (65), (66), and (68), we deduce the existence of $\varphi$, $\boldsymbol{A}, \theta$, and $\boldsymbol{\mu}$ satisfying Equations (28)-(34). This ends the proof of Theorem 2.

Remark 4 In this work, we have stated an existence result of weak solutions to system (17)-(24). Nevertheless, the assumption (26) on the electric conductivity is so weak that we cannot assure that the Radon measure is $\boldsymbol{\mu}=0$. 


\section{Acknowledgements}

This research has been partially supported by the Project MTM2010-16401 of Ministerio de Economía y Competitivad with the participation of the FEDER, and Consejería de Educación y Ciencia de la Junta de Andalucía, research group FQM-315.

\section{References}

[1] C. Amrouche, C. Bernardi, M. Dauge, and V. Girault, Vector potentials in three-dimensional non-smooth domains, Math. Methods Appl. Sci. 21 (1998), pp. 823-864.

[2] A. Bermúdez, J. Bullón, F. Pena, and P. Salgado, A numerical method for transient simulation of metallurgical compound electrodes, Finite Elem. Anal. Des. 39 (2003), pp. 283-299.

[3] A. Bermúdez, D. Gómez, M.C. Muñiz, and P. Salgado, Transient numerical simulation of a thermoelectrical problem in cylindrical induction heating furnaces, Adv. Comput. Math. 26 (2007), pp. 39-62.

[4] L. Boccardo and T. Gallouët, Non-linear elliptic and parabolic equations involving measure data, J. Funct. Anal. 87 (1989), pp. 149-169.

[5] K. Chełminski, D. Hömberg, and D. Kern, On a Thermomechanical Model of Phase Transitions in Steel, WIAS preprint 1125, Berlin, 2007.

[6] S. Clain, Analyse mathématique et numérique d'un modèle de chauffage par induction, Ph.D. thesis N. 1240, Laussan, EPFL, 1994.

[7] J.M. Díaz Moreno, C. García Vázquez, M.T. González Montesinos, and F. Ortegón Gallego, Un modelo para la descripción de las transiciones de fases en una barra de acero, Actas XX Congreso de Ecuaciones Diferenciales y Aplicaciones/X Congreso de Matemática Aplicada, Sevilla, 2007, pp. 24-28.

[8] J.M. Díaz Moreno, C. García Vázquez, M.T. González Montesinos, and F. Ortegón Gallego, Numerical simulation of an induction-conduction model arising in steel hardening, World Congress on Engineering 2009, Lecture Notes in Engineering and Computer Science, Vol. II, 2009, pp. 1251-1255.

[9] J.M. Díaz Moreno, C. García Vázquez, M.T. González Montesinos, and F. Ortegón Gallego, Analysis and numerical simulation of an induction-conduction model arising in steel heat treating, J. Comput. Appl. Math. 236(12) (2012), pp. 3007-3015.

[10] J.M. Díaz Moreno, C. García Vázquez, M.T. González Montesinos, F. Ortegón Gallego, and G. Viglialoro, Mathematical modeling of heat treatment for a steering rack including mechanical effects, to appear in J. Numer. Math.

[11] J. Fuhrmann, D. Hömberg, and M. Uhle, Numerical simulation of induction hardening of steel, COMPEL 18(3) (1999), pp. 482-493.

[12] M.T. González Montesinos and F. Ortegón Gallego, On an induction-conduction PDE system in harmonic regime, to appear.

[13] D. Hömberg, A mathematical model for induction hardening including mechanical effects, Nonlinear Anal. RWA 5 (2004), pp. 55-90.

[14] D. Hömberg and J. Sokolowski, Optimal shape design of inductor coils for surface hardening, SIAM J. Control Optim. 42(3) (2003), pp. 1087-1117.

[15] D. Hömberg and W. Weiss, PID control of laser surface hardening of steel, IEEE Trans. Control Syst. Technol. 14(5) (2006), pp. 896-904.

[16] F.J. Pena Brage, Contribución al modelado matemático de algunos problemas en la metalurgia del silicio, Ph.D. thesis, Universidade de Santiago de Compostela, 2003.

[17] J. Simon, Compact sets in the space $L^{p}(0, T ; B)$, Ann. Mat. Pura Appl. 146(4) (1987), pp. 65-96.

[18] H.M. Yin, Regularity of weak solution to Maxwell's equations and applications to microwave heating, J. Differential Equations 200 (2004), pp. 137-161. 